\title{
Drug resistance profiles and trends in drug- resistant tuberculosis at a major hospital in Guizhou Province of China
}

\author{
Yuanbo Lan ${ }^{1, *}$ \\ Yuqin $\mathrm{Li}^{1}$,* \\ Ling Chen' \\ Jianyong Zhang' \\ Hong Zhang ${ }^{1,2}$ \\ 'Department of Respiratory Medicine, \\ Affiliated Hospital of Zunyi Medical \\ College, Zunyi, Guizhou 563003.

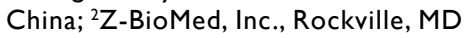 \\ 20855, USA
}

*These authors contributed equally to

\author{
this work
}

This article was published in the following Dove Medical Press journal: Infection and Drug Resistance
Objectives: Tuberculosis (TB) is the leading cause of death from infectious diseases in the world, with an estimated 1.6 million deaths from TB in 2017. The objectives of this study were to determine drug resistance profiles of Mycobacterium tuberculosis clinical isolates and to analyze the trends in drug-resistant and multidrug-resistant tuberculosis (MDR-TB) from 2008 to 2015 at a major hospital in Guizhou, a high-TB burden and resource-limited province of China. Patients and methods: A total of 462 clinical isolates were collected from patients with pulmonary TB during the period from January 2013 to December 2015 and used for determining drug resistance profiles against four first-line and six second-line anti-TB drugs, and the results were compared with those of two previous studies.

Results: Exactly $4.4 \%$ of new and $44.1 \%$ of previously treated TB cases were MDR/rifampicinresistant TB (RR-TB), which were higher than the 2017 global average numbers of $3.5 \%$ and $18 \%$, respectively. There were many drug-resistant patterns among MDR-TB isolates and most of them were resistant to three or four anti-TB drugs. The trends in drug-resistant and MDR-TB declined at the hospital from 2008 to 2015.

Conclusion: Results of this study show that the prevalence of MDR/RR-TB at a major hospital in Guizhou is higher than the global average and confirm the existence of heterogeneity in drug resistance patterns of MDR-TB isolates. Additionally, three practical measures have played an important role in the overall decline of MDR-TB at the hospital from 2008 to 2015.

Keywords: Mycobacterium tuberculosis, anti-TB drugs, drug resistance patterns, trends

\section{Introduction}

Antibiotic-resistant bacteria constitute a heavy and growing economic burden on all nations of the world, and the rise of antimicrobial resistance poses a global threat to the effective prevention and treatment of an ever-increasing range of infections caused by bacteria, parasites, viruses and fungi. ${ }^{1}$ Tuberculosis (TB) is the tenth leading cause of death and the leading cause of death from infectious diseases in the world, and drug-resistant TB can occur when appropriate anti-TB drugs are not prescribed or are taken incorrectly in both the public health and hospital systems. Globally, in 2017, an estimated 558,000 people developed rifampicin-resistant TB (RR-TB), of whom 82\% had multidrug-resistant TB (MDR-TB). Also, in 2017, an estimated $3.5 \%$ of new and $18 \%$ of previously treated TB cases had MDR/RR-TB, and almost half $(47 \%)$ of these MDR/RR-TB cases were from three high TB burden countries: India (24\%), China (13\%) and the Russian Federation (10\%). In addition, 127 countries reported extensively drug-resistant TB (XDR-TB) cases to the WHO 
by the end of 2017, and the average proportion of MDRTB cases with XDR-TB was $8.5 \%$, which was higher than that in $2016(6.2 \%){ }^{2}$

China is one of the 14 countries that are in all three highburden country lists for TB, TB/HIV and MDR-TB defined by WHO during the period 2016-2020. ${ }^{2}$ Data from the 2017 survey of China showed that $7.1 \%$ of new and $24 \%$ of previously treated cases of TB were MDR/RR-TB, and $4.8 \%$ of MDR/RR-TB cases were XDR-TB. However, the treatment success rate for MDR/RR-TB patients who started on the second-line treatment in China in 2015 was only $41 \% .^{2}$ The prevalence of drug-resistant TB and MDR-TB in Zunyi, Guizhou Province of China, was higher than the national average, and there were different drug resistance patterns among MDR-TB clinical isolates. ${ }^{3,4}$ Due to insufficient laboratory capacities and infrastructures, the treatment of drug-resistant TB in rural areas and some cities of Guizhou Province is mainly empirical, which makes the management of drug-resistant TB, especially MDR-TB, more difficult and challenging in a province with high TB burden and limited resources.

The currently recommended treatment for cases of drugsusceptible TB is a 6-month regimen of four first-line anti-TB drugs. For the treatment of RR-TB or MDR-TB patients with documented or suspected susceptibility to the drugs to be used, a longer treatment regimen with at least five effective anti-TB drugs during the intensive phase is recommended by WHO as the standardized regimen. ${ }^{5}$ However, treatment options for patients with MDR-TB are limited and expensive in some low- and middle-income countries, and some patients may have serious adverse reactions from second-line anti-TB drugs. ${ }^{5}$ To minimize adverse reactions and improve treatment outcomes of patients with drug-resistant TB, drug susceptibility testing (DST) against first- and second-line anti-TB drugs has been routinely performed at the Affiliated Hospital of Zunyi Medical College for years.

It was reported previously that the population rates of MDR-TB declined in some of the 53 Member States in the WHO European Region, such as Estonia, Latvia and Germany, but increased in other Member States, such as the UK, Sweden and Tomsk Oblasts of the Russian Federation, between 1997 and 2012. ${ }^{6}$ Twenty years of global surveillance of anti-TB drug resistance showed decreasing trends in MDRTB among new TB cases in Australia, Estonia, Hong Kong, Israel, Latvia, Portugal and the USA and increasing trends in Austria, Finland, Sweden and the UK, which were associated with immigration. ${ }^{7}$ However, limited data on trends of MDR-TB in African and Asian countries were available in
2016 due to the challenges in repeating surveys at regular intervals or establishing continuous surveillance. ${ }^{?}$

Results from a recent study on the 10-year trends in drug-resistant TB at a clinical TB center in Beijing, China showed that the percentage of MDR-TB among all TB patients increased gradually from $13.1 \%$ in 2005 to $19.3 \%$ in 2008 , but decreased every year after $2008 .{ }^{8}$ Among 18,310 in-patients recruited from the 30 provinces of China for the study, only 67 patients were from Guizhou Province, ${ }^{8}$ indicating necessity to study the trends of drug-resistant TB in Guizhou, the province with the second highest TB prevalence among the 31 provinces that participated in the 2010 national survey of TB in China. ${ }^{9}$ The objectives of this study were to determine the drug resistance profiles of Mycobacterium tuberculosis clinical isolates collected from January 2013 to December 2015 and to analyze the trends of drug-resistant M. tuberculosis clinical isolates collected from 2008 to 2015 at a major hospital in Guizhou Province.

\section{Patients and methods}

\section{Study population and clinical isolates of}

\section{M. tuberculosis}

This study was conducted at the Affiliated Hospital of Zunyi Medical College, a 3,500-bed tertiary general hospital and one of the provincial medical centers/major hospitals in Guizhou Province. The Department of Respiratory Medicine is specialized in the treatment of patients with MDR-TB and provides services and critical care to thousands of patients with TB, COPD, asthma and other respiratory diseases every month. As part of routine hospital laboratory procedures, a total of 462 clinical isolates of M. tuberculosis were consecutively collected from the sputum and bronchoscope brush specimens of TB patients from January 2013 to December 2015 . The collected clinical specimens were processed by following the procedures recommended by WHO and cultured on Löwenstein-Jensen solid slants, and grown colonies were identified to the species level. ${ }^{10}$

\section{Drug susceptibility testing}

M. tuberculosis culture and DST were conducted at the Laboratory of Respiratory Medicine in the hospital, which was certified by the Chinese Center for Disease Control and Prevention (China CDC) to conduct DST on TB isolates. Sputum specimens collected from patients with suspected pulmonary TB were cultured according to the operational procedures recommended by WHO in a biologic safety cabinet to avoid potential contaminations. ${ }^{10}$ One to three milliliters of 
samples were added to testing tubes containing two volumes of $4 \%$ sodium hydroxide $(\mathrm{NaOH})$ solution, and the mixtures were shaken briefly and allowed to stand at room temperature for 15 minutes with occasional shaking. After that, $0.1 \mathrm{~mL}$ of samples was transferred onto Löwenstein-Jensen solid slants and incubated for 8 weeks at $37^{\circ} \mathrm{C}$. Cultures were examined 72 hours after inoculation to check whether the liquid was completely evaporated and the caps were tightened. Thereafter, the cultures were examined once a week until the end of the eighth week, and results were recorded every time.

The proportion method on Löwenstein-Jensen media was used for conducting the DST against four first-line (rifampicin, isoniazid, streptomycin and ethambutol) and six second-line (gatifloxacin, levofloxacin, moxifloxacin, amikacin, capreomycin and kanamycin) anti-TB drugs purchased from Sigma-Aldrich (St Louis, MO, USA), Enzo Life Sciences (Farmingdale, NY, USA) or LKT Laboratories (St Paul, MN, USA). ${ }^{11,12}$ The critical concentrations (drug concentrations necessary to eliminate susceptible strains of M. tuberculosis) and the critical proportions (the proportion of a population of $M$. tuberculosis needed to be resistant to a drug in order for that strain to be interpreted as resistant) of four first-line and six second-line anti-TB drugs for DST using the proportion method in Löwenstein-Jensen media are listed in Table 1. The standard M. tuberculosis strain $\mathrm{H} 37 \mathrm{Rv}$ was obtained from the China CDC and used as the quality control.

\section{Analysis of trends in drug-resistant TB}

For the goal of achieving better clinical management and more efficient control of MDR-TB in Guizhou Province of China, we analyzed the trends of drug-resistant $M$. tuberculosis clinical isolates collected from 2008 to 2015 at a major hospital in Guizhou Province by comparing the results of this study with those of two previous studies using M. tuberculosis clinical isolates collected at the same hospital from 2008 to 2010 and from 2011 to $2012 .^{3,4}$

\section{Results \\ Patients' characteristics}

During the period from January 2013 to December 2015, M. tuberculosis clinical isolates were collected one isolate per patient from 462 patients with active pulmonary TB at the hospital. Among 462 TB patients, 319 (69\%) were new and 143 (31\%) were previously treated; also, the proportion of male $(290,62.8 \%)$ to female $(172,37.2 \%)$ patients was similar to the national estimated TB incidence in China for male $(67.5 \%)$ and female $(32.5 \%)$ patients reported to WHO in $2017 .{ }^{2}$ Most patients $(445,96.3 \%)$ were from three age groups: $16-39$ years $(146,31.6 \%), 40-59(166,35.9 \%)$ and $60-79(133,28.8 \%)$. Only six patients $(1.3 \%)$ were younger than 15 years and eleven patients $(2.4 \%)$ were older than 80 years (Table 2).

\section{Prevalence of drug-resistant TB}

We analyzed $462 M$. tuberculosis clinical isolates for their drug susceptibility profiles against four first-line anti-TB drugs, three second-line later-generation fluoroquinolones (gatifloxacin, levofloxacin and moxifloxacin) and three injectable agents (amikacin, capreomycin and kanamycin) during this study period. Among 319 new TB cases, 28 $(8.8 \%)$ were resistant to isoniazid, $14(4.4 \%)$ to rifampicin, 27 $(8.5 \%)$ to streptomycin, $13(4.1 \%)$ to ethambutol, $11(3.4 \%)$ were MDR-TB and 14 (4.4\%) were MDR/RR-TB (Table 2). However, the prevalence of drug resistance against the four first-line anti-TB drugs in previously treated TB cases was much higher than that in the new TB cases: $68(47.6 \%), 63$ (44.1\%), 52 (36.4\%), 46 (32.2\%), 55 (38.5\%) and $63(44.1 \%)$

Table I Critical concentrations and critical proportions of anti-TB drugs for DST using the proportion method in Löwenstein-Jensen media

\begin{tabular}{|l|l|l|l|}
\hline Drug & Solvent & Critical concentration $(\mu \mathrm{g} / \mathrm{mL})$ & Critical proportion (\%) \\
\hline Rifampicin & DMF & 40 & $\mathrm{I}$ \\
Isoniazid & DW & 0.2 & $\mathrm{I}$ \\
Ethambutol & DW & 2 & $\mathrm{I}$ \\
Streptomycin & DW & 4 & $\mathrm{I}$ \\
Gatifloxacin & $0.1 \mathrm{~mol} / \mathrm{L} \mathrm{HCl}$ & 2 & $\mathrm{I}$ \\
Levofloxacin & DW & 2 & $\mathrm{I}$ \\
Moxifloxacin & DW & 2 & $\mathrm{I}$ \\
Amikacin & DW & 30 & $\mathrm{I}$ \\
Capreomycin & DW & 40 & $\mathrm{I}$ \\
Kanamycin & DW & 30 & $\mathrm{I}$ \\
\hline
\end{tabular}

Abbreviations: DMF, dimethylformamide; DST, drug susceptibility testing; DW, sterile distilled water; TB, tuberculosis. 
Table 2 Demographic characteristics of TB patients and drug resistance profiles of Mycobacterium tuberculosis clinical isolates to ten anti-TB drugs

\begin{tabular}{|c|c|c|c|c|c|c|}
\hline \multirow[t]{2}{*}{$\begin{array}{l}\text { Characteristics and } \\
\text { drug resistance }\end{array}$} & \multicolumn{2}{|c|}{ New cases $(n=3 \mid 9)$} & \multicolumn{2}{|c|}{$\begin{array}{l}\text { Previously treated } \\
\text { cases }(n=143)\end{array}$} & \multicolumn{2}{|c|}{ Total cases $(n=462)$} \\
\hline & $n$ & $\%$ & $n$ & $\%$ & $n$ & $\%$ \\
\hline \multicolumn{7}{|l|}{ Gender } \\
\hline Male & 192 & 60.2 & 98 & 68.5 & 290 & 62.8 \\
\hline Female & 127 & 39.8 & 45 & 31.5 & 172 & 37.2 \\
\hline \multicolumn{7}{|l|}{ Age (years) } \\
\hline$\leq 15$ & 4 & 1.3 & 2 & 1.4 & 6 & 1.3 \\
\hline $16-39$ & 106 & 33.2 & 40 & 27.9 & 146 & 31.6 \\
\hline $40-59$ & 110 & 34.5 & 56 & 39.2 & 166 & 35.9 \\
\hline $60-79$ & 92 & 28.8 & 41 & 28.7 & 133 & 28.8 \\
\hline$\geq 80$ & 7 & 2.2 & 4 & 2.8 & 11 & 2.4 \\
\hline \multirow{2}{*}{\multicolumn{7}{|c|}{$\begin{array}{l}\text { Resistance to } \\
\text { first-line anti- } \\
\text { TB drugs }\end{array}$}} \\
\hline & & & & & & \\
\hline Rifampicin & 14 & 4.4 & 63 & 44.1 & 77 & 16.7 \\
\hline Isoniazid & 28 & 8.8 & 68 & 47.6 & 96 & 20.8 \\
\hline Ethambutol & 13 & 4.1 & 46 & 32.2 & 59 & 12.8 \\
\hline Streptomycin & 27 & 8.5 & 52 & 36.4 & 79 & 17.1 \\
\hline MDR-TB & 11 & 3.4 & 55 & 38.5 & 66 & 14.3 \\
\hline \multirow{3}{*}{\multicolumn{7}{|c|}{$\begin{array}{l}\text { Resistance to } \\
\text { second-line } \\
\text { anti-TB drugs }\end{array}$}} \\
\hline & & & & & & \\
\hline & & & & & & \\
\hline \multicolumn{7}{|l|}{ Fluoroquinolones } \\
\hline Gatifloxacin & 10 & 3.1 & 13 & 9.1 & 23 & 5.0 \\
\hline Levofloxacin & 12 & 3.8 & 24 & 16.8 & 36 & 7.8 \\
\hline Moxifloxacin & 9 & 2.8 & 17 & 11.9 & 26 & 5.6 \\
\hline \multicolumn{7}{|l|}{ Injectables } \\
\hline Amikacin & 5 & 1.6 & 11 & 7.7 & 16 & 3.5 \\
\hline Capreomycin & 11 & 3.4 & 7 & 4.9 & 18 & 3.9 \\
\hline Kanamycin & 4 & 1.3 & 9 & 6.3 & 13 & 2.8 \\
\hline Pre-XDR-TB & 2 & 0.6 & 14 & 9.8 & 16 & 3.5 \\
\hline XDR-TB & 1 & 0.3 & 7 & 4.9 & 8 & 1.7 \\
\hline
\end{tabular}

Abbreviations: MDR-TB, multidrug-resistant TB; TB, tuberculosis; XDR-TB, extensively drug resistant TB.

of previously treated TB cases were resistant to isoniazid, rifampicin, streptomycin, ethambutol, MDR-TB and MDR/ RR-TB, respectively (Table 2). Overall, 96 (20.8\%), 79 (17.1\%), 77 (16.7\%), 59 (12.8\%), 66 (14.3\%) and 77 (16.7\%) M. tuberculosis clinical isolates were resistant to isoniazid, streptomycin, rifampicin, ethambutol, MDR-TB and MDR/ RR-TB, respectively (Table 2).

The DST results against six second-line anti-TB drugs showed that the resistance rates for 319 new TB cases were $3.8 \%$ (levofloxacin), 3.4\% (capreomycin), 3.1\% (gatifloxacin), $2.8 \%$ (moxifloxacin), $1.6 \%$ (amikacin) and 1.3\% (kanamycin). Only two new cases $(0.6 \%)$ of TB were pre-XDR and one $(0.3 \%)$ was XDR (Table 2). The resistance rates for 143 previously treated TB cases were $16.8 \%$ (levofloxacin), $11.9 \%$ (moxifloxacin), 9.1\% (gatifloxacin), 6.3\% (kanamy- cin), 7.7\% (amikacin), 3.4\% (capreomycin), 9.8\% (pre-XDR) and $4.9 \%$ (XDR), as shown in Table 2.

\section{Drug resistance profiles of MDR-TB clinical isolates}

Among 66 MDR-TB clinical isolates, 9 (13.6\%) were resistant to just rifampicin and isoniazid, 17 (25.8\%) to three first-line anti-TB drugs and 16 (24.3\%) were resistant to four first-line anti-TB drugs. The DST results of 66 MDR-TB isolates against six second-line anti-TB drugs showed that 18 (27.3\%) isolates were resistant to levofloxacin, 14 (21.2\%) isolates to moxifloxacin, 10 (15.2\%) isolates to gatifloxacin, 9 (13.6\%) isolates to amikacin, $5(7.6 \%)$ isolates to kanamycin and $4(6.1 \%)$ isolates were resistant to capreomycin (Table 3). In addition, 6 (9.1\%) isolates were resistant to five drugs, 
Table 3 Drug resistance patterns of 66 MDR-, pre-XDR- and XDR-TB clinical isolates from a major hospital in Guizhou Province of China

\begin{tabular}{|c|c|c|c|c|c|c|}
\hline \multirow[t]{2}{*}{ Drug resistance patterns } & \multicolumn{2}{|c|}{$\begin{array}{l}\text { New cases } \\
(n=I I)\end{array}$} & \multicolumn{2}{|c|}{$\begin{array}{l}\text { Previously treated } \\
\text { cases }(n=55)\end{array}$} & \multicolumn{2}{|c|}{$\begin{array}{l}\text { Total cases } \\
(n=66)\end{array}$} \\
\hline & $\mathbf{n}$ & $\%$ & $\mathbf{n}$ & $\%$ & $\mathbf{n}$ & $\%$ \\
\hline \multicolumn{7}{|l|}{ MDR (no. of anti-TB drugs) } \\
\hline RIF, INH (2) & 3 & 27.3 & 6 & 10.9 & 9 & 13.6 \\
\hline RIF, INH, EMB (3) & I & 9.1 & 7 & 12.7 & 8 & 12.1 \\
\hline RIF, INH, STR (3) & I & 9.1 & 8 & 14.6 & 9 & 13.6 \\
\hline RIF, INH, EMB, STR (4) & 3 & 27.3 & 13 & 23.6 & 16 & 24.3 \\
\hline Subtotal & 8 & 72.7 & 34 & 61.8 & 42 & 63.6 \\
\hline \multicolumn{7}{|l|}{ Pre-XDR (no. of anti-TB drugs) } \\
\hline RIF, INH, EMB, LFX (4) & 0 & 0.0 & I & 1.8 & 1 & 1.5 \\
\hline RIF, INH, EMB, MOX (4) & 0 & 0.0 & 1 & 1.8 & 1 & 1.5 \\
\hline RIF, INH, LFX, MOX (4) & I & 9.1 & 0 & 0.0 & I & 1.5 \\
\hline RIF, INH, EMB, LFX, MOX (5) & 0 & 0.0 & 1 & 1.8 & 1 & 1.5 \\
\hline RIF, INH, EMB, STR, LFX (5) & I & 9.1 & 1 & 1.8 & 2 & 3.1 \\
\hline RIF, INH, EMB, STR, MOX (5) & 0 & 0.0 & 1 & 1.8 & 1 & 1.5 \\
\hline RIF, INH, STR, AMK, KAN (5) & 0 & 0.0 & 1 & 1.8 & I & 1.5 \\
\hline RIF, INH, EMB, AMK, CAP, KAN (6) & 0 & 0.0 & 1 & 1.8 & I & 1.5 \\
\hline RIF, INH, EMB, STR, GFX, LFX (6) & 0 & 0.0 & 2 & 3.7 & 2 & 3.1 \\
\hline RIF, INH, EMB, STR, AMK, CAP, KAN (7) & 0 & 0.0 & 1 & 1.8 & I & 1.5 \\
\hline RIF, INH, EMB, STR, GFX, LFX, MOX (7) & 0 & 0.0 & 4 & 7.4 & 4 & 6.1 \\
\hline Subtotal & 2 & 18.2 & 14 & 25.5 & 16 & 24.3 \\
\hline \multicolumn{7}{|l|}{ XDR (no. of anti-TB drugs) } \\
\hline RIF, INH, STR, LFX, KAN (5) & 0 & 0.0 & 1 & 1.8 & I & 1.5 \\
\hline RIF, INH, STR, LFX, MOX, AMK (6) & 0 & 0.0 & I & 1.8 & 1 & 1.5 \\
\hline RIF, INH, EMB, LFX, MOX, AMK, CAP, KAN (8) & 0 & 0.0 & 1 & 1.8 & 1 & 1.5 \\
\hline RIF, INH, EMB, STR, GFX, LFX, MOX, AMK (8) & 0 & 0.0 & 3 & 5.5 & 3 & 4.5 \\
\hline RIF, INH, EMB, STR, GFX, LFX, MOX, AMK, CAP (9) & I & 9.1 & 1 & 1.8 & I & 1.5 \\
\hline Subtotal & I & 9.1 & 7 & 12.7 & 8 & 12.1 \\
\hline Total & 11 & 100.0 & 55 & 100.0 & 66 & 100.0 \\
\hline
\end{tabular}

Abbreviations: AMK, amikacin; CAP, capreomycin; EMB, ethambutol; GFX, gatifloxacin; INH, isoniazid; KAN, kanamycin; LFX, levofloxacin; MDR, multidrug resistant; MOX, moxifloxacin; pre-XDR, MDR with additional resistance to a fluoroquinolone or a second-line injectable drug; RIF, rifampicin; STR, streptomycin; TB, tuberculosis; $X D R$, extensively drug resistant.

$4(6.1 \%)$ isolates to six drugs, $5(7.6 \%)$ isolates to seven drugs, $4(6.1 \%)$ isolates to eight drugs, $1(1.5 \%)$ isolate to nine drugs, $16(24.3 \%)$ isolates were pre-XDR-TB and 8 (12.1\%) isolates were XDR-TB (Figure 1).

\section{Trends in drug-resistant TB}

For new TB cases, the drug resistance rates for rifampicin, isoniazid, ethambutol and streptomycin decreased during the period 2008-2015 (Figure 2). For previously treated TB cases, drug resistance rates for the four first-line anti-TB drugs also decreased when the results of this study were compared with those from 2008 to 2010 (Table 4; Figure 2). Overall, the drug resistance rates for the four first-line anti-TB drugs and MDR-TB declined from 2008 to 2015 with the exception of ethambutol during the period 2011-2012 (Table 4; Figure 2).

\section{Discussion}

During the study period, M. tuberculosis clinical isolates were obtained from 462 patients with active pulmonary $\mathrm{TB}$ at

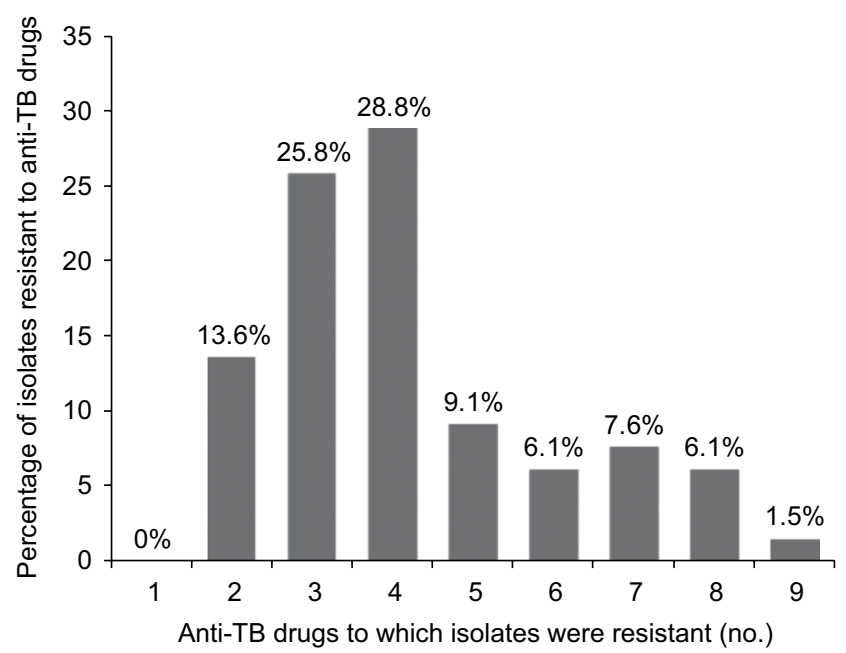

Figure I Drug resistance patterns.

Notes: Sixty-six MDR-, pre-XDR- and XDR-TB clinical isolates were tested for drug susceptibility against four first-line (rifampicin, isoniazid, streptomycin and ethambutol) and six second-line (gatifloxacin, levofloxacin, moxifloxacin, amikacin, capreomycin and kanamycin) anti-TB drugs. The heterogeneity in drug resistance patterns of MDR-TB isolates is displayed as a percentage of isolates resistant to a different number of anti-TB drugs.

Abbreviations: MDR-TB, multidrug-resistant TB; TB, tuberculosis; XDR-TB, extensively drug resistant TB. 


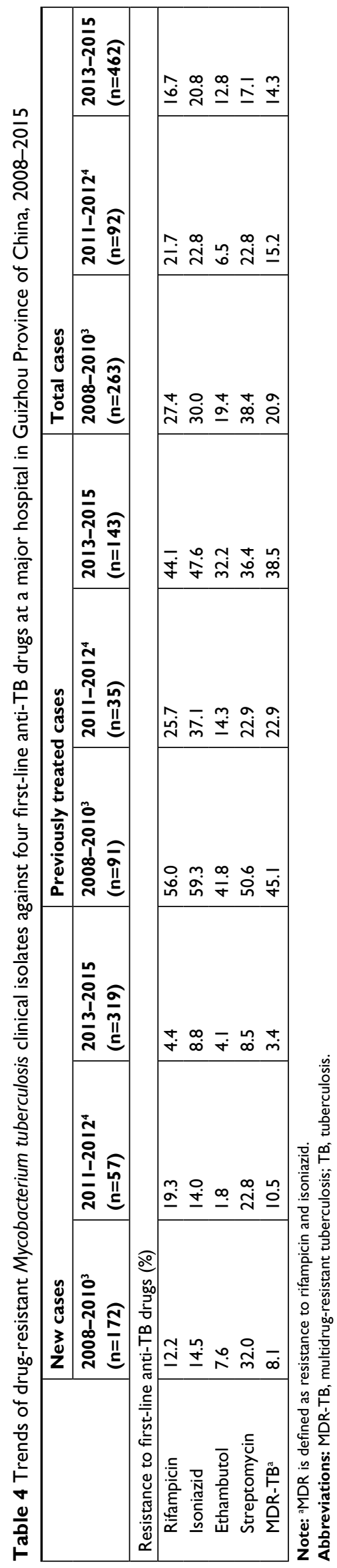

the hospital. Among them, the proportion of male to female patients was $290(62.8 \%)$ to $172(37.2 \%)$, similar to the national estimated TB incidence in China for male (67.4\%) and female (32.6\%) patients. ${ }^{2}$ Regarding the age groups of the patient population, 166 (35.9\%) were from the $40-59$ age group, 146 (31.6\%) from the 16-39 age group and 133 (28.8\%) were from the 16-39 age group (Table 2). It is clear that over two-thirds of TB patients $(312,67.5 \%)$ were between the ages of 16 and 59 , and not many patients were very young (only 6 were $<15$ years) or very old ( 11 were $\geq 80$ years).

The DST results against four first-line anti-TB drugs showed that $4.4 \%$ of new and $44.1 \%$ of previously treated cases were MDR/RR-TB, which were higher than the global average numbers in 2017 (an estimated 3.5\% of new and 18\% of previously treated cases had MDR/RR-TB), ${ }^{2}$ but lower than the combined data from 53 Member States in the WHO European Region (an estimated $15.7 \%$ of new and $45.3 \%$ of previously treated TB cases had MDR-TB). ${ }^{6}$ Twenty years of global surveillance of anti-TB drug resistance showed that the percentage of new TB cases with MDR-TB remained stable, at $3 \%$ or lower, in most parts of the world. Nevertheless, the highest percentages of new TB cases with MDR-TB were reported in 2014 in countries in Eastern Europe and central Asia, such as Belarus (34.1\%), Estonia (19.5\%) and Ukraine (24.0\%). ${ }^{7}$ The highest percentages of previously treated TB cases with MDR-TB were reported in 2014 in Belarus (69.1\%), Estonia (62.1\%) and Tajikistan (52.2\%). ${ }^{7}$

Results from the current study also showed that $12.1 \%$ $(8 / 66)$ of MDR-TB cases were XDR-TB, which was much higher than the average numbers of MDR-TB cases with XDR-TB in China (4.8\%), in the entire world (6.2\%), in 53 Member States in the WHO European Region (11.4\%) and in a tertiary care TB hospital in Jiangxi Province of China (10.6\%), but lower than those at a TB hospital in Beijing (14.9\%) and in South Africa (63.3\%)..$^{2,713-15}$ It is worth noting that $24.2 \%(16 / 66)$ of MDR-TB cases were pre-XDR-TB, which is twice the number of XDR-TB (12.1\%). Overall, more than one-third of the MDR-TB cases (24/66, 36.3\%) were either pre-XDR-TB or XDR-TB (Table 2), which makes successful treatment of MDR-TB patients more difficult in Guizhou Province of China. Therefore, it is very important to design the optimal combination of second-line anti-TB drugs for previously treated and MDR-TB patients in Guizhou Province based on the DST results.

The order of preference for fluoroquinolones recommended by WHO to treat MDR-TB is as follows: high-dose levofloxacin, moxifloxacin and gatifloxacin. ${ }^{5}$ However, the orders of resistance for the three fluoroquinolones among 


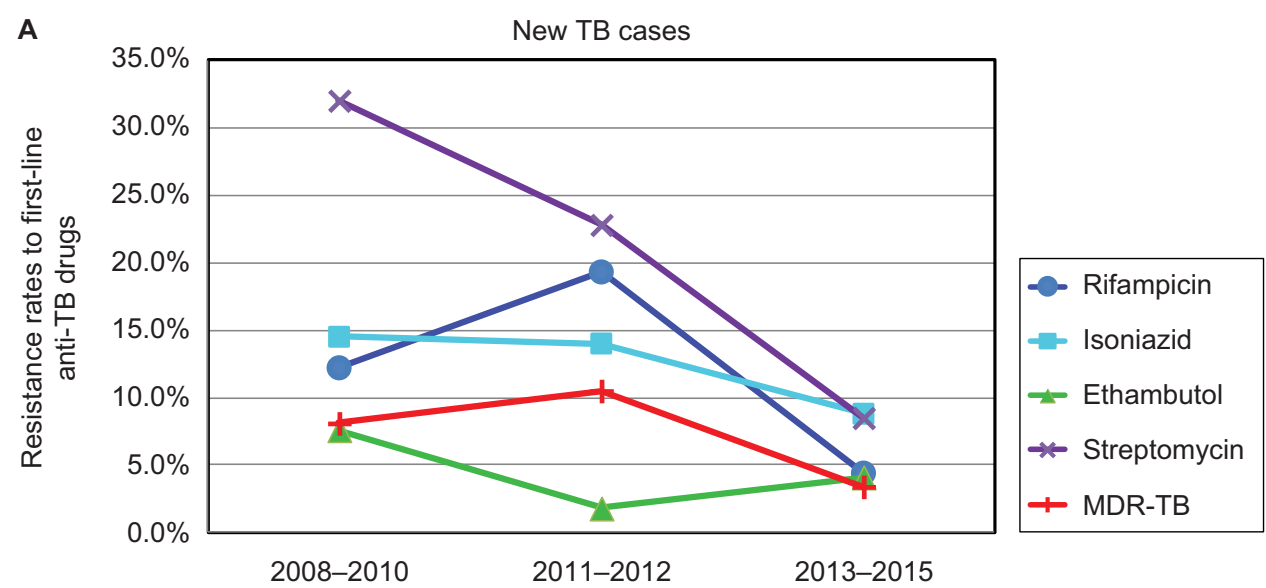

B Previously treated TB cases
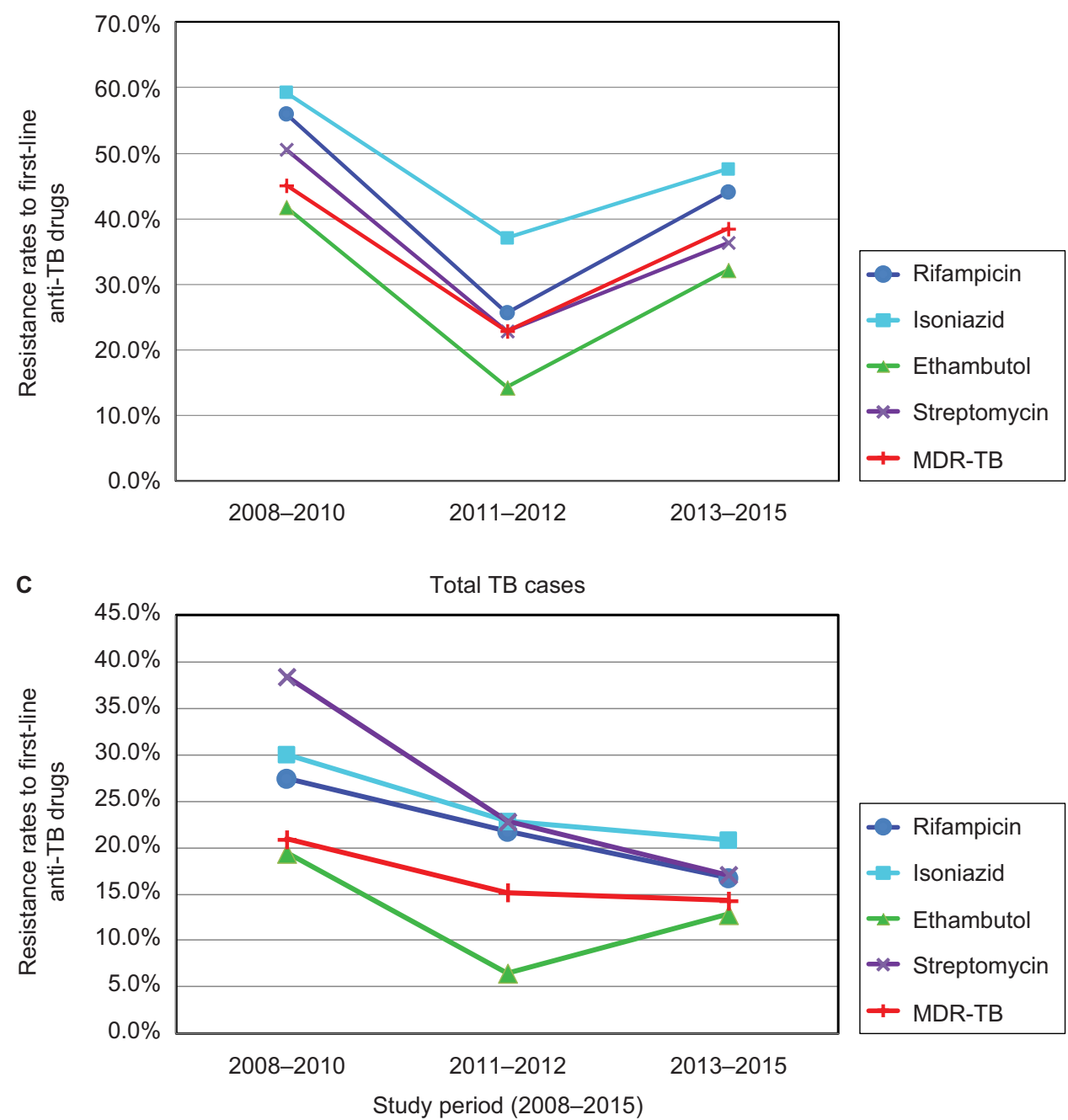

Figure 2 Trends of drug resistance rates to first-line anti-TB drugs.

Notes: Trends of drug resistance rates for four first-line anti-TB drugs (rifampicin, isoniazid, streptomycin and ethambutol) and MDR-TB in new TB cases (A), previously treated TB cases (B) and total TB cases (C) at a major hospital in Guizhou Province of China during the study period 2008-2015.

Abbreviations: MDR, multidrug resistant; TB, tuberculosis.

143 previously treated TB cases (Table 2) and $66 \mathrm{MDR}-\mathrm{TB}$ cases (Table 3) were found to be the same as the order of preference: $16.8 \%$ and $27.3 \%$ to levofloxacin, $11.9 \%$ and $21.2 \%$ to moxifloxacin and $9.1 \%$ and $15.2 \%$ for gatifloxa- cin. Among 66 MDR-TB clinical isolates, 9 (13.6\%) were resistant to two, $17(25.8 \%)$ to three, 19 (28.8\%) to four, 6 (9.1\%) to five, $4(6.1 \%)$ to six, $5(7.6 \%)$ to seven, 4 (6.1\%) to eight and $1(1.5 \%)$ was resistant to nine anti-TB drugs 
(Figure 1). These results confirm the existence of many drugresistant patterns among MDR-TB clinical isolates, and that most MDR-TB isolates are resistant to three or four anti-TB drugs. We believe that the heterogeneity in drug resistance patterns of MDR-TB isolates is caused by mutational heterogeneity in mycobacterial genes associated with resistance to anti-TB drugs. As suggested by WHO, in settings in which laboratory capacity for DST to second-line anti-TB drugs is not available, such as rural areas of Guizhou Province of China, treatment decisions would need to be guided by the likelihood of resistance to these drugs based on the patient's clinical history and recent surveillance data. ${ }^{5}$

To analyze the trends of drug-resistant TB during the period 2008-2015 at a major hospital in Guizhou Province, the DST results against the four first-line drugs of this study were compared with the results of two previous studies using M. tuberculosis clinical isolates collected from 2008 to $2010^{3}$ and from 2011 to $2012 .{ }^{4}$ It is obvious that drug resistance rates for rifampicin, isoniazid, ethambutol and streptomycin declined from the year 2008 to 2015 in new TB cases (Figure 2). For previously treated TB cases, the drug resistance rates for the four first-line anti-TB drugs also declined when compared to those from 2008 to 2010 (Table 4; Figure 2). Overall, the drug resistance rates for MDR-TB among total TB patients declined from $20.9 \%$ in $2008-2010$ to $14.3 \%$ in 2013-2015 (Table 4; Figure 2). It was reported that the percentage of MDR among all TB cases in Zhejiang Province of China decreased from $8.6 \%$ in 1999 to $5.1 \%$ in 2004, which indicated that TB control programs in Zhejiang Province were working efficiently. ${ }^{16}$ In another report, the percentage of MDR among all TB cases in greater metropolitan Mumbai, India decreased from $39.4 \%$ in the period $2005-2007$ to $27.8 \%$ in 2011-2013, while the proportion of those with XDR-TB increased from $6.1 \%$ to $10.6 \%$ during the same time period. ${ }^{17}$ However, the trend in drug resistance against the four first-line anti-TB drugs showed a significant increase between 1996 and 2004 at a tertiary hospital in Iran, which indicated inefficient TB control programs and improper antiTB drug use during those years. ${ }^{18}$

We believe that three practical measures have played an important role in the overall decline of MDR-TB among all the TB patients at the hospital. First, we had provided examination, anti-TB drugs, TB education and psychological support to patients with drug-resistant and MDR-TB without charging any fee, through funding such as "National Science and Technology Major Projects" and "Global Fund" between 2010 and 2013. Second, we carried out many continuing education courses, and training sessions during major con- ferences, in which well-known TB experts from Beijing and Shanghai were invited to give lectures to physicians, nurses, technicians and health care providers from this hospital as well as hospitals in the surrounding cities, counties and other parts of Guizhou on prevention, diagnosis, and treatment of drug-resistant TB. Third, we established strong coalition with other departments at the hospital, relevant social organizations, local hospitals and health care providers, and the media to enhance public awareness of TB and educate the general public as well as specific population groups, such as the illiterate and elderly from rural areas of western Guizhou, and to create a favorable social atmosphere for better management and control of TB.

In order to ensure cure without relapse, reduce the high TB mortality rate and prevent the spread of drug-resistant TB, especially MDR-TB, new therapeutic approaches toward improving TB treatment outcomes were summarized in two recent review articles. ${ }^{19,20}$ These new approaches included discovery of new chemical entity with a novel mechanism of action, repurposing of old drugs, the use of novel drug delivery systems and development of host-directed therapies.

\section{Conclusion}

Results from the current study show that the prevalence of MDR/RR-TB at this major hospital in Guizhou Province is higher than the average number in the world and confirm the existence of heterogeneity in drug resistance patterns of MDR-TB isolates. In addition, the proportion of MDR-TB among new and previously treated TB patients declined during the period 2008-2015, indicating that practical measures for TB control at this major hospital have been effective. The practical measures include the project support, high-quality training for medical staff and enhanced public awareness of TB prevention and control. This study indicates that it is necessary to conduct routine DST in TB patients from highburden and resource-limited areas such as Guizhou Province of China in order to reduce the global burden of TB.

\section{Acknowledgments}

We thank all the clinical and laboratory staff from the Department of Respiratory Medicine, Affiliated Hospital of Zunyi Medical College for their contribution in collecting clinical data and specimens from patients, culture of Mycobacterium tuberculosis clinical isolates and performing drug susceptibility testing for this study. This study was supported by the National Natural Science Foundation of China (grant nos 81760003, 81360002 and 81160003), the second $2011 \mathrm{Col}-$ laborative Innovation Center for TB Prevention and Cure in 
Guizhou Province (Incubation Project), the Key Laboratory of Infectious Disease \& Biosafety, Provincial Department of Education, the Global Fund Projects for TB Standardized Diagnosis and Treatment (CHN-S10-G14-T) and the fourth Talented Individual Base for Infectious Disease Prevention and Control in Guizhou Province. The sponsors played no role in the collection, analysis and interpretation of data; in writing the manuscript; and in the decision to submit the article for publication.

\section{Author contributions}

All authors contributed to data analysis, drafting or revising the article, gave final approval of the version to be published, and agree to be accountable for all aspects of the work.

\section{Disclosure}

HZ is employed by and has shares in Z-BioMed, Inc., which is involved in infectious disease research. The authors report no other conflicts of interest in this work.

\section{References}

1. World Health Organization. Antimicrobial Resistance. Geneva: World Health Organization; 2018. Available from: http://www.who.int/newsroom/fact-sheets/detail/antimicrobial-resistance. Accessed September 15, 2018.

2. World Health Organization. Global Tuberculosis Report 2018. Geneva: World Health Organization; 2018. Available from: http://www.who.int/ tb/publications/global_report/en. Accessed December 3, 2018.

3. Chen L, Li N, Liu M, Zhang J, Zhang H. High prevalence of multidrugresistant tuberculosis in Zunyi, Guizhou Province of China. JAntimicrob Chemother. 2011;66(10):2435-2437.

4. Li N, Liao X, Chen L, Wang J, Liu M, Zhang H. Antibiotic susceptibility patterns of Mycobacterium tuberculosis isolates from Guizhou Province of China against 13 antituberculosis drugs. Microb Drug Resist. 2015;21(3):292-296.

5. World Health Organization. WHO Treatment Guidelines for DrugResistant Tuberculosis, 2016 Update. Geneva, Switzerland: World Health Organization; 2016. Available from: http://apps.who.int/medicinedocs/en/d/Js23097en/. Accessed September 15, 2018.

6. Zignol M, Dara M, Dean AS, et al. Drug-resistant tuberculosis in the WHO European Region: an analysis of surveillance data. Drug Resist Updat. 2013;16(6):108-115.
7. Zignol M, Dean AS, Falzon D, et al. Twenty years of global surveillance of antituberculosis-drug resistance. $N$ Engl J Med. 2016;375(11):1081-1089.

8. Liao S, Cai C, Huo FM, et al. Trends in drug-resistant tuberculosis in China: data from a clinical tuberculosis centre. Int J Tuberc Lung Dis. 2017;21(9):990-995.

9. Technical Guidance Group of the Fifth National TB Epidemiological Survey, Office of the Fifth National TB Epidemiological Survey. 2010 National Tuberculosis Epidemiological Survey in China. Chin J Antituberculosis. 2012;34:485-508. Chinese.

10. World Health Organization. Laboratory Services in Tuberculosis Control. Part III: Culture. Geneva: World Health Organization; 1998. Available from: http://apps.who.int/iris/bitstream/handle/10665/65942/ WHO_TB_98.258_(part3).pdf;jsessionid=64EF54513AD4144A520B 24B4E3DFCBA5? sequence=3. Accessed December 3, 2018.

11. Challenge TB, TB Care I, TB CAP and partners. Standard operating procedures for culture DST and molecular resistance testing. Laboratory Tools, Guidelines and Manuals. Available from: http://www.challengetb. org/library/lab. Accessed December 3, 2018.

12. World Health Organization. Guidelines for drug susceptibility testing for second-line anti-tuberculosis drugs for DOTS-PLUS. WHO/ CDS/TB/2001.288. Available from: http://apps.who.int/iris/bitstream/ handle $/ 10665 / 83807 /$ ? sequence $=1$. Available from: December 3, 2018.

13. Yuan X, Zhang T, Kawakami K, et al. Genotyping and clinical characteristics of multidrug and extensively drug-resistant tuberculosis in a tertiary care tuberculosis hospital in China. BMC Infect Dis. 2013;13:315.

14. Wang D, Yang C, Kuang T, et al. Prevalence of multidrug and extensively drug-resistant tuberculosis in Beijing, China: a hospital-based retrospective study. Jpn J Infect Dis. 2010;63(5):368-371.

15. Shah NS, Richardson J, Moodley P, et al. Increasing drug resistance in extensively drug-resistant tuberculosis, South Africa. Emerg Infect Dis. 2011;17(3):510-513.

16. Huang Y, Wu Q, Xu S, et al. Laboratory-based surveillance of extensively drug-resistant tuberculosis in eastern China. Microb Drug Resist. 2017;23(2):236-240.

17. Dalal A, Pawaskar A, Das M, et al. Resistance patterns among multidrugresistant tuberculosis patients in greater metropolitan Mumbai: trends over time. PLoS One. 2015;10(1):e0116798.

18. Shamaei M, Marjani M, Chitsaz E, et al. First-line anti-tuberculosis drug resistance patterns and trends at the national TB referral center in Iran - eight years of surveillance. Int J Infect Dis. 2009;13(5):e236-e240.

19. Patil K, Bagade S, Bonde S, Sharma S, Saraogi G. Recent therapeutic approaches for the management of tuberculosis: challenges and opportunities. Biomed Pharmacother. 2018;99:735-745.

20. du Plessis N, Kotze LA, Leukes V, Walzl G. Translational potential of therapeutics targeting regulatory myeloid cells in tuberculosis. Front Cell Infect Microbiol. 2018;8:332.
Infection and Drug Resistance

\section{Publish your work in this journal}

Infection and Drug Resistance is an international, peer-reviewed openaccess journal that focuses on the optimal treatment of infection (bacterial, fungal and viral) and the development and institution of preventive strategies to minimize the development and spread of resistance. The journal is specifically concerned with the epidemiology of antibiotic

\section{Dovepress}

resistance and the mechanisms of resistance development and diffusion in both hospitals and the community. The manuscript management system is completely online and includes a very quick and fair peerreview system, which is all easy to use. Visit http://www.dovepress.com/ testimonials.php to read real quotes from published authors. 